\title{
Werden Sie Fachkraft für Interventionelle Radiologie (FIR)!
}

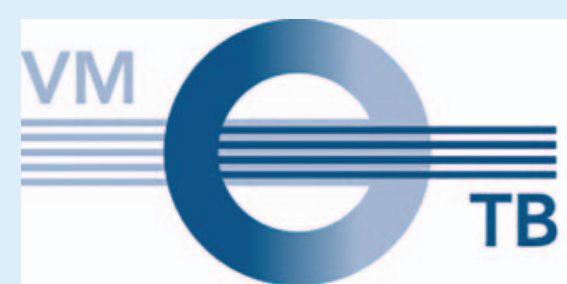

Liebe Kolleginnen und Kollegen,

die Anforderungen an das Personal bei interventionellen Eingriffen werden zunehmend höher. Die VMTB hat in Zusammenarbeit mit der DRG und der DeGIR ein Curriculum für die Fachkraft Interventionelle Radiologie ins Leben gerufen.

Wir laden Sie herzlich zum 6. zertifizierten Kurs „Fachkraft Interventionelle Radiologie
(FIR)“ nach Regensburg ein. Dieser Kurs richtet sich an alle, die in interventionellen Arbeitsbereichen tätig sind, sich weiterbilden und zertifizieren lassen wollen. Es werden in 4 Tagen theoretisch und praktisch die Bereiche Strahlenschutz, Materialkunde, gesetzliche Regelungen, Hygiene, Personal, Leitlinien und Entwicklungen vermittelt.

Jedes Modul schließt mit einer Prüfung ab. Die Modulzertifikate werden von der DRGGeschäftsstelle versandt. Mit Bestehen der Module 1-3 kann das Zertifikat „Fachkraft Interventionelle Radiologie“ bei der DRG beantragt werden.

Begleitet wird die Veranstaltung durch ein kleines Rahmenprogramm. Hier haben die
Teilnehmer/Teilnehmerinnen die Möglichkeit, Erfahrungen auszutauschen und Netzwerke aufzubauen.

Wir würden uns freuen, Sie bei uns in Regensburg begrüßen zu dürfen!

Ihre

Claus Becker, Franziska Fleischmann, Prof. Dr. Christian Stroszczynski

\section{Die Termine:}

13.-17. Juli 2021

19.-22. Juli 2021

12.-15. Oktober 2021

18.-21. Oktober 2021 